\title{
TALLER DE COACHING PARA MEJORAR LA SATISFACCIÓN DE LAS RELACIONES INTERPERSONALES ENTRE PROFESORES
}

\author{
COACHING WORKSHOP TO IMPROVE THE \\ SATISFACTION OF INTERPERSONAL RELATIONS \\ BETWEEN TEACHERS
}

Mónica Sánchez Sanssoni Universidad Católica San Pablo, Arequipa, Perú

\section{Resumen:}

El presente estudio de corte experimental tiene por finalidad valorar el efecto de un taller de coaching en la satisfacción las relaciones interpersonales de los profesores de la institución educativa de Arequipa. Para ello se trabajó con un taller de diez sesiones y se aplicó el Cuestionario de Satisfacción en las Relaciones Interpersonales en el Trabajo. El instrumento fue suministrado en dos momentos (antes y después de las sesiones de coaching), a 45 profesores que forman parte de nuestra muestra. Las aplicaciones pre y post test indican que entre una y otra hay diferencias significativas en la satisfacción de las relaciones interpersonales en el trabajo, lo cual da cuenta de la eficiencia del taller de coaching. No se han registrado diferencias significativas en la variable de estudio, según el sexo, el cargo, el área de trabajo, la ocupación ni la profesión de los docentes.

Palabras clave: Coaching, relaciones interpersonales, gestión educativa.

\section{Summary:}

The present experimental study aims to assess the impact of a workshop of coaching in interpersonal relations satisfaction of teachers from Arequipa. To do this, we worked with a ten-session workshop and applied the Satisfaction Questionnaire in Interpersonal 
Relations at Work. The instrument was applied in two moments (before and after the coaching sessions), to 45 teachers who are part of our sample. The pre and post test applications indicate that between one and the other there are significant differences in the satisfaction of interpersonal relations at work, which gives an account of the efficiency of the workshop of coaching. There were no significant differences in the study variable, according to gender, position, area of work, occupation or profession.

Key words: Coaching, interpersonal relationships, educational management.

\section{Introducción}

El término "coaching" se origina en Hungría entre el siglo XV y el siglo XVI, en la ciudad de Kocs, donde habían unos carruajes llamados "Kosci szeker" utilizados para trasladarse con fines turísticos. La palabra coaching proviene entonces del término "carruaje de Kocs” (Lárez, 20o8). Sin embargo, a partir de los '8o, en parte por la obra de Beverly Showers, se empieza a utilizar en los contextos educativos (Jiménez, 2012). Ahora bien, como dice Lárez (2008) el coaching se utilizó profusamente en el ámbito deportivo, para luego aplicarse a los escenarios organizacionales, de consejería, educativos y diversos campos profesionales. Por ello, dicen Nava y Mena (2012) que la palabra coach tiene dos significados: entrenador y carruaje. Para otros el coaching es un término polisémico, que tiene múltiples significados, usos y aplicaciones, en distintos ámbitos y con diversos fines (Moreno et al., 2013).

Hoy se puede distinguir el coaching personal, ejecutivo, empresarial, profesional, deportivo y educacional (O'Connors y Lages, 2010). En ese sentido, se debe diferenciar la labor del coach, del entrenador, el tutor, el mentor y el terapeuta; aunque todos ellos comparten algunas características comunes propias del coaching. Para ello debemos partir señalando qué es el coaching. O'Connors y Lages (2010) indican que el coaching es una actividad orientada al cambio en función del planteamiento de determinadas metas. Entonces, las metas van a variar en función del campo en el que se aplique el coaching, pero siempre va a girar en torno al cambio, el aprendizaje, el liderazgo, la reflexión y las emociones positivas.

Para Nava y Mena (2012) el coaching es un "proceso de acompañamiento a un individuo o un grupo, para ayudarlos a profundizar el conocimiento de sí mismos y guiarlos en el desarrollo de nuevas acciones con el objetivo de mejorar su desempeño personal y profesional para obtener óptimos resultados" (p. 118).

En ese sentido, aunque el coach es un motivador no es un entrenador personal, aunque utiliza técnicas terapéuticas no es un terapeuta, aunque promueve las destrezas y capacidades de los coachees no es un mentor (ya que el mentor siempre sabe más que el discípulo, el coach en cambio puede no ser un experto en el campo en el que se desarrolla el coachee), aunque dirige y orienta a las personas no es un tutor.

Ciertamente es complejo definir el coaching, por ello, para algunas personas, 
el coaching presenta diversos obstáculos para ser objeto de estudio, sin embargo, en los últimos años, el coaching ha generado diversas investigaciones, sobre todo en los campos organizacional y educativo. Muchas de estas investigaciones son ubicables en tesis de pre y postgrado en diversas áreas del saber, pero es menos común encontrar artículos académicos sobre el coaching.

En la presente investigación la autora se avoca al coaching educativo, que es más fácil de definir y que cuenta con abundante investigación, sobre todo en temas relativos a la investigación (Moreno, Álvarez e Inojosa, 2013), la formación de profesores (Jiménez, 2012b) y el desarrollo, profesional (Jiménez, 2012a). Nuestra investigación se circunscribe al campo del desarrollo profesional, con énfasis en las relaciones interpersonales.

Según Hirsch, el coaching educativo es un proceso continuo que se enfoca en las destrezas, competencias y técnicas, siendo su finalidad, el aumentar la efectividad de la enseñanza y ofrecer aprendizaje individualizado para el profesor. Para Reiss, el coaching es un proceso que promueve el cambio transformacional. Para Knight el coaching educativo es un enfoque que ofrece apoyo a los profesores, para obtener nuevas formas de pensar y practicar sus enseñanzas a partir de la reflexión conjunta, la discusión y la exploración (Wise y Avendaño, 2013).

El coaching educativo por tanto, abarca tanto una filosofía como una metodología. A nivel filosófico, el coaching se basa en una manera de comprender y percibir el mundo de manera optimista, poniendo mucho interés en las virtudes humanas, la autorrealización y la gestión eficaz de las propias potencialidades humanas. A nivel metodológico, su rasgo más esencial es la práctica concreta, pues aunque se nutre de diversas técnicas, perspectivas, enfoques, actividades, procedimientos, etc.; aplica técnicas activas de aprendizaje y formación. Por ejemplo, puede haber un coaching sistémico (Ayuso et al., 2010), un coaching basado en la programación lingüística (O’Connors y Lages, 2010), un coaching cognitivo (Nava y Mena, 2012), un coaching fenomenológico (Moreno et al., 2013), un coaching basado en enfoques socioculturales (Jiménez, 2012b), o en las neurociencias (Escribano, 2009), o un coaching basado en la psicología positiva (Seligman, 2006); pero los rasgos mencionados, estarán siempre presentes.

En América Latina el coaching es una práctica reciente como tal, pero si consideramos las implicancias del coaching, podemos decir que el liderazgo educativo sería una de las formas en las que el coaching se venía aplicando desde hace varios años atrás (Barrientos, 2015). Un estudio de Beganza y Escribano (2004), reportó que en España las organizaciones implementan principalmente el coaching individual (56\%); tiene una duración promedio de 1 a 9 meses; aplican la metodología de equipos de trabajo (75\%); como características más valoradas se señalan la experiencia y las habilidades interpersonales del coach, así como el compromiso, la rigurosidad y su discreción. Además, los planes de coaching son coordinados por el área de Recursos Humanos (49\%) y lo que más afecta el coaching es la falta de apoyo de la dirección. También se reportó que en la mayoría de 
las organizaciones, no se evalúa el impacto del coaching (69\%), y se considera que su principal aporte es el desarrollo de capacidades para el trabajo (44\%).

A nivel educativo, podemos decir que el coaching se focaliza en cuestiones como el aprendizaje, el liderazgo, la innovación, los valores, las emociones positivas, la inteligencia emocional y las habilidades sociales. Además se aplica con la finalidad de mejorar la escuela, en diferentes instancias y focalizándose en todos sus actores, según los métodos, planes y objetivos del coach.

En ese sentido, son tantos los actores que inciden en la enseñanza, que es más recomendable aplicar el coaching educativo con metas claras y objetivos precisos. Para ello un diagnóstico previo es de gran ayuda (O'Connors y Lages, 2010). En ese diagnóstico conviene abarcar factores macro y micro de la escuela, como los que refiere Arias (2008). Este autor indica que a nivel macro, la educación comprende el sistema educativo, las políticas de educación, los factores socioeconómicos y otros de tipo contextual con respecto a las escuelas. A nivel micro, se tienen la escuela, los aspectos organizacionales, el cuerpo docente, el aula y los currículos. Siguiendo esta perspectiva, podemos decir que el coaching está diseñado para incidir en los aspectos micro. Por ejemplo en el estudio de Gorrochotegui-Martell, Vicente-Mendoza y Torres-Escobar (2014) se aplicó un programa de coaching dirigido al equipo directivo de una escuela de la capital de Chile, para lo cual se evaluó el clima escolar según los padres, los profesores y los alumnos. Se encontró que tras la aplicación del programa, los padres y alumnos reportaron un clima escolar más satisfactorio, cosa que no ocurrió con el profesorado.

Este diagnóstico, nos permite, antes de aplicar el coaching, valorar los aspectos deficitarios de la gestión educativa, ya sea con acento pedagógico o administrativo. En el primer caso, el coaching se centra en la práctica pedagógica del docente con la finalidad de mejorar el aprendizaje de los estudiantes, mientras que en el segundo caso, se avoca a diversas cuestiones de orden institucional.

A nivel pedagógico, el coaching se parece mucho a la actividad tutorial. Por ello, autores como Lárez (2008) señalan que el tutor debe ser un coach para los alumnos. Siguiendo ese razonamiento, el coach tutor requiere de las siguientes habilidades: Saber escuchar y atender a los estudiantes, ofrecer disponibilidad de ayuda, saber hacer su trabajo docente y de coach, ser competente profesionalmente, tener buen ánimo y una actitud mental positiva, usar una metodología de trabajo precisa.

A nivel de institucional, interesa que el coach se focalice en diversos aspectos organizacionales relacionados con la gestión educativa y que directa o indirectamente, inciden en los procesos de enseñanza-aprendizaje. En ese sentido, un estudio realizado en Arequipa, encontró por ejemplo, que la percepción que tenían los estudiantes de nivel secundario de una institución educativa pública sobre la calidad de la gestión, se relacionaba con el rendimiento académico que tenían en la escuela, aunque se obtuvieron coeficientes de correlación variables en función del año de estudios (Arias, Zavala y Bernilla, 
2014). Esto da evidencias de cómo algunos aspectos organizacionales como la calidad de la gestión educativa inciden en el aprendizaje de los estudiantes. En consecuencia, el coaching puede ayudar a mejorar ciertos procesos educativos y por ende generar buenos resultados, lo que se refleja en la percepción de los actores implicados, sean éstos, estudiantes, profesores, trabajadores o padres de familia. El estudio de Gorrochotegui-Martell, Vicente-Mendoza y Torres-Escobar (2014) es otro ejemplo de ello.

Precisamente, desde una perspectiva organizacional es que se han documentado mayor cantidad de estudios sobre el coaching. Por ejemplo, en la investigación de Lárez (2008) se aplicó el coaching a la gestión de la investigación, siguiendo los siguientes criterios: La responsabilidad del trabajo de investigación es de los autores, el uso de la mayéutica, la facilitación de recursos y herramientas necesarias para hacer investigación, fomentar valores y una cultura de investigación, tener conocimiento de la labor investigativa, brindar acompañamiento durante el proceso de investigación, tener una visión sabia, los rasgos de personalidad del coach.

A nivel organizacional, se pueden distinguir varios modelos, dentro de los cuales, a decir de Arqueros (2009) sobresalen cuatro, en los que se pone énfasis en el liderazgo y la empatía, así como en el trabajo en equipo y la comunicación.

El modelo de Saporito (1996) se aplica en ejecutivos para cuestiones de consultoría y se divide en cuatro escenarios. Primero se define el contexto, luego se da un proceso de guía para el desarrollo de personas en 360 grados. Después se planifica el desarrollo en base al feedback y al liderazgo; para finalmente realizar el coaching ejecutivo a partir del desarrollo de experiencias. En el modelo de Kirkpatrick (1994) se trabaja más la evaluación, también en cuatro niveles: reacción, aprendizaje, comportamiento y resultados. En el modelo de Passmore (2007) se trabaja el coaching en tres niveles: comportamental, cognitivo e inconsciente. Propone además, cuatro corrientes de cambio o flujo: Necesidad de los coaches de trabajar con lo que pueden ver, que viene a ser el comportamiento, necesidad de trabajar con lo que pueden escuchar, que tiene que ver con la cognición, necesidad de trabajar con el inconsciente, necesidad de trabajar sistémicamente. En el modelo de Kilburg (2001) se pone énfasis en la adherencia, y para lograr ello, se tienen ocho componentes clave: Comportamiento de los clientes, compromiso del coach, características de los problemas y cuestiones de los clientes, estructura del contenido del proceso de coaching, relación cliente-coach, calidad de las intervenciones de coaching, protocolo de adherencia, marco organizacional del cliente-coach.

Por otro lado, dado que el coaching en el escenario organizacional, sirve para adaptarse a los cambios, liderar y generar innovación, se debe orientar hacia el cambio de mentalidad. Para ello, el coaching cognitivo, es muy adecuado, ya que al cambiar la mentalidad de las personas se concentra en la ética, el liderazgo, la asertividad, la reflexión, entre otras habilidades cognitivas (Nava y Mena, 2012).

La metodología más usada empero, es la de trabajo en equipo. El coaching en equipos 
crea una base fundamental para el autoconocimiento y facilita el crecimiento del grupo. Mira a la persona como un miembro inmerso en un sistema o una red vincular. El equipo es por tanto un sistema dinámico y activo, con su propia cultura y reglas de funcionamiento. El coaching en equipos está orientado a la consecución de objetivos colectivos y se fundamenta generalmente en el enfoque sistémico (Ayuso, 2010).

El coaching en equipos se sustenta en la enseñanza cooperativa, y recibe diversos nombres, amén de que puede trabajarse desde diversas modalidades: coaching entre iguales, apoyo colaborativo, amigo crítico, etc. En el estudio de Rodríguez et al. (2011), el 81\% valoró la técnica de amigo crítico de manera positiva y el $75 \%$ señaló que las sesiones de trabajo fueron motivadoras. Por ello, puede decirse que el coaching entre iguales es un potente instrumento de desarrollo profesional, además de ser una importante estrategia para el aprendizaje en equipos, que se enrique con la propia experiencia y con las aportaciones de los compañeros, permitiendo enfocarse en una perspectiva cooperativa de trabajo (Rodríguez et al., 2011).

En ese sentido, las escuelas son comunidades de aprendizaje que se caracterizan por: 1) fomentar las relaciones colaborativas entre el profesorado; 2) la construcción de una cultura pedagógica sobre valores, principios y prácticas; y 3) procesos de investigación sobre la práctica, que conllevan análisis y revisión crítica de los hechos y métodos (Jiménez, 2012b).

Ahora bien, en Lima, Manuel Fernández (2002) ha investigado el síndrome de burnout en los profesores de primaria, y ha encontrado que muchos maestros se encuentran estresados y agobiados por diversas condiciones de trabajo. En nuestra localidad, un se ha encontrado que los profesores de colegios nacionales presentan mayores niveles de despersonalización los que se manifiesta en el trato indiferente hacia sus estudiantes, mientras que en los profesores de colegios privados, se observan mayores niveles de baja realización personal, lo que se explica por la labor más demandante que deben realizar (Arias y Jiménez, 2013a).

Otro estudio encontró que altos niveles de estrés se relacionan con la creatividad en los profesores (Arias, Montes y Masías, 2014), por lo que se infiere que se trata de un estrés positivo, también conocido como eustrés, mientras que el estrés negativo es el que se conoce como distrés. Sin embargo, a nivel de relaciones interpersonales, Arias y Jiménez (2013b) ha reportado que las relaciones humanas de los trabajadores de mypes de Arequipa son negativas. Para el caso de los profesores, sus hallazgos indican que una gran mayoría de profesores presenta relaciones de tipo inseguro, egoísta y agresivo (Arias, 2013a).

En cuanto a las relaciones interpersonales, Portugal (200o), las define como las vinculaciones que se van conformando en la vida real entre los individuos que sienten y piensan. Hernández (2004) dice que las relaciones interpersonales son el conjunto de interacciones humanas mediadas por los factores personales y sociales. En ese sentido, las relaciones interpersonales se ubican entre el individuo y lo social, por tanto tienen una gran relevancia, pues 
comunican dos aspectos de la vida humana que son determinantes para el desarrollo de la humanidad (Hochman, 2010).

En ese sentido, no son pocos los estudios que señalan que así como las relaciones interpersonales afectan positivamente el clima organizacional (Orduño, 20o6), el clima organizacional también puede afectar las relaciones interpersonales (Rota, Reynolds Zamasi, 2012). En este caso se evidencia que las condiciones de trabajo desfavorables, pueden afectar las relaciones interpersonales y por ende la eficacia del trabajo (Ripollo et al., 2004).

En nuestro país, los estudios sobre relaciones interpersonales en contextos laborales, son escasos, pero sí es posible encontrar investigaciones sobre los valores interpersonales en contextos educativos (Matalinares et al., 2009). Las relaciones interpersonales son importantes porque nos comunican acerca de las vinculaciones sociales con otros, y el tejido de interacciones que se establece tiene un fuerte impacto en las maneras de convivencia que se desarrollan entre las personas. De este modo, las relaciones interpersonales, según sean favorables o desfavorables, pueden ser catalizadores de nuestro mundo interno en el trabajo, o bien ser obstáculos que limitan el trabajo del docente (Lugo, 2004).

Por ejemplo, en el estudio de Arias (2013b) se encontró que en ocho empresas de Arequipa, aunque el clima organizacional fue negativo debido básicamente al bajo reconocimiento a la labor de los trabajadores y las altas demandas laborales, pero que la cohesión de los trabajadores y las buenas relaciones humanas en el trabajo, fueron un factor protector de la salud laboral y les permitía compensar otras deficiencias en la organización del trabajo.

Ahora bien, las relaciones humanas tienen que ver con el liderazgo, la inteligencia emocional y las habilidades sociales. En ese sentido, las habilidades sociales son fortalezas que nos ayudan a relacionarnos con los demás, de manera adecuada y asertiva (Mamani, 2006). Para ello, la inteligencia emocional, nos permite gestionar nuestras emociones y las de los demás, a partir de la correcta lectura de los afectos humanos (Goleman, 200o). Las relaciones interpersonales también se relacionan con el liderazgo por cuanto, van surgiendo de manera espontánea roles de liderazgo entre las personas, según sus habilidades y las situaciones (Jay, 2004).

Como dice Fingerman (1980) las relaciones interpersonales pueden verse afectadas por diversos factores, por ejemplo la percepción de justicia. Fernández (2009) por ejemplo, nos dice que hay diversas formas de justicia en el trabajo, distributiva, procedimental, interactiva, etc. Lo importante es tener en cuenta que las relaciones entre los trabajadores deben ser equilibradas y que los dirigentes deben velar siempre por brindar un trato equitativo sin favoritismos. Por ejemplo, en el trabajo de Díaz L. (2005) se menciona que les relaciones interpersonales pueden ser afectadas por el rol que juegan los sindicatos. Precisamente, Arias (2013a) concluyó en su estudio, que las pésimas relaciones humanas halladas entre los profesores del sector público de Arequipa, podrían deberse al discurso reaccionario de los dirigentes sindicales, que lejos de promover la reconciliación y 
una actitud proactiva en el trabajo educativo, genera desunión y enconos cada vez más notables.

Por otro lado, las relaciones interpersonales en tanto se objetivan a través de las interacciones humanas (Bisquerra, 2003) y por lo tanto pueden ser susceptibles de medición objetiva y cuantificarse (Díaz A., 2005), pero hay que tener en cuenta dos criterios: uno conceptual o teórico y otro estadístico o tecnológico. En el primer caso, existen diversos modelos teóricos que nos permiten entender las relaciones humanas. Hay por ello teorías socio-críticas, situacionales, organizacionales, sistémicas, etc.

En el segundo caso, el fundamento estadístico, muchas veces no permite confirmar los modelos teóricos propuestos, en parte por fallas metodológicas, y en otras ocasiones debido a que la teoría no se condice con la realidad. En ese sentido, algo similar ha ocurrido con el coaching, con el que las relaciones interpersonales comparten entre otras cosas, sus relaciones con el liderazgo y la inteligencia emocional (Jay, 2004). En ese sentido, es importante abordar la vida afectiva de los profesores, pues de ello depende su correcto desenvolvimiento y por ende el aprendizaje de los estudiantes (Pérez, 2001).

El coaching puede promover el aprendizaje de las personas, ya que les enseña a aprender a aprender. Uno de los aspectos más importantes del coaching es precisamente ese. En ese sentido, el coaching es una aproximación estratégica y sistemática a la mejora del aprendizaje, y aunque puede aplicarse a los estudiantes, es más frecuente su uso en la formación de profesores, ya sea que usen modelos puros o mixtos (Jiménez, 2012b). Nava y Mena (2012) señalan que el coaching es en el fondo una modalidad de aprendizaje, pues el coaching facilita que las personas de adapten a los cambios fácilmente, por ello es ideal para los contextos organizacionales, ya que las organizaciones del siglo XXI cambian constantemente, debido a la introducción de la tecnología en el trabajo y a las nuevas fórmulas empresariales que privilegian al trabajador en lugar de las instancias burocráticas como ocurría en la primera mitad del siglo XX. La globalización de los mercados es otro factor que ha hecho posible este cambio de visión en las organizaciones.

El coaching también promueve los valores y el liderazgo, por ello Nava y Mena (2012) dicen que es un sistema de motivación y liderazgo que activa los valores personales y los compromisos del ser humano. Dentro de los valores que se trabajan en el coaching, se tienen como primordiales, el involucramiento en el trabajo y la orientación de metas que conducen a la mejora constante y a una política de exigencia continua, o si se quiere de autoperfeccionamiento. En ese sentido, desde el punto de vista axiológico el coaching enfatiza el compromiso y la responsabilidad para cumplir con funciones definidas (Moreno, Álvarez e Inojosa, 2013).

El coaching también promueve las emociones positivas. Según Alles (2009), el coach debe inyectar optimismo y motivación a las personas a la vez que se focaliza en la visión-misión del trabajo. Por eso, la dimensión psicológica, conjuga los aspectos motivacionales y emocionales (Moreno, Álvarez e Inojosa, 2013). Nosotros tuvimos 
la oportunidad de aplicar un taller basado en el amor y la ternura en estudiantes de primero de primaria que mostraban conducta indisciplinada, y conseguimos resultados notables en valores tales como la responsabilidad, la justicia y (Sánchez, 2015).

Esta nueva tendencia dentro de la psicología y la pedagogía científica surge en el 2000, y ha generado abundante investigación que hasta la fecha señala que los factores que más generan bienestar en el hombre son: Autoaceptación, crecimiento personal, propósito en la vida, dominio del entorno, autonomía, relaciones positivas.

El coaching promueve todas estas características en las personas, pero además de ello, desde un ángulo pedagógico, abarca factores educacionales abarcan tales como la reflexión, la creatividad, el pensamiento crítico, etc. (Moreno, Álvarez e Inojosa, 2013). A nivel profesional el coaching propone un entrenamiento en el desarrollo de las competencias técnicas que inciden profundamente en las personas (Jiménez, 2012b).

De acuerdo con Nava y Mena (2012) los principios del coaching son: Respeto por la persona, autonomía, método personalizado, consideración de las emociones, reflexión sobre el sentido de los actos, deseo de cambio, feedback constante, construcción de confianza, mutualidad, empatía y confidencialidad.

Es así que el coach mediante diversas estrategias de aprendizaje y pautas de conducta, establece una orientación hacia el carácter individual y la consecución de acción. Está además estructurado en cuatro etapas según Jiménez (2012b): conversaciones reflexivas, feedback positivo, proceso constructivo, apoyo emocional. Para Maisel (2005) se tienen las siguientes etapas: Identificación, selección y formación de coach, toma de contacto y creación de condiciones, delimitación de situaciones problemas, sesiones de diseño y planificación colaborativa de procesos de enseñanza.-aprendizaje, implementación de procesos de enseñanza-aprendizaje, sesiones de reflexión guiada sobre las intervenciones educativas.

Para O'Connor y Lages (2010), las etapas iniciales del coaching son esenciales porque se definen las metas y se hace el diagnóstico inicial. Además se formula el plan de acción, se establecen los métodos y técnicas para su implementación y se conoce a los participantes, coachees o clientes. Asimismo, se pueden mencionar una diversidad de técnicas usadas para el coaching, muchas de las cuales se usan en el mentoring, la terapia y el entrenamiento personal. Entre estas técnicas se tiene: la pausa, el parafraseo, y el sondeo, que son técnicas conversacionales que retroalimentan a la persona. También se tiene el reflejo de emoción, el reflejo de contenido, y las preguntas circulares que favorecen la interacción y la escucha activa de los participantes. Las técnicas de la estatua, el role playing, la silla vacía, etc. Son técnicas que nos permiten realizar diversas dinámicas y juegos transaccionales con la finalidad de promover la empatía que es base de la confianza (Berne, 1998).

Todas estas técnicas aplicadas al campo de la formación docente, aumentan en el profesorado, la conciencia profesional y la 
transferencia de aprendizajes a la práctica (Jiménez, 2012b). Por ello, la metodología del coaching aplicada al medio educativo es de gran utilidad, porque desarrolla competencias para un desempeño óptimo y reconoce la necesidad de lograr que los actores educativos acepten invertir todo su talento en la educación (Moreno, Álvarez e Inojosa, 2013).

En ese sentido, el desarrollo profesional docente abarca ocho principios, según Jiménez (2012): Objetivos del profesorado, aprendizaje contextualizado resolución colaborativa de problemas, apoyo suficiente el proceso, información rica y rigurosa, oportunidades para la comprensión teórica, es procesual, es continuo.

Finalmente, tenemos que en el ámbito educativo, las modalidades de coaching son a nivel de supervisión, el mentoring, a nivel de formación el team coaching, educational coaching, instructional coaching, cognitive coaching y el content-focused coaching. El más usado en la educación es el team coaching que usualmente se acompaña del coaching cognitivo (Jiménez, 2012a).

Así, nuestra investigación se basa en los siguientes objetivos: 1) Determinar en qué medida la aplicación de un taller de coaching mejora la satisfacción de las relaciones interpersonales de los docentes de una institución educativa de Arequipa, y 2) Determinar las diferencias del nivel de satisfacción de las relaciones interpersonales en función del sexo, grado de instrucción, profesión, ocupación, área de trabajo y cargo de los docentes, después de aplicar el taller de coaching.

\section{Material y Métodos}

Dado que lo que se pretende con esta investigación es valorar los efectos de un taller de coaching, se trata de un estudio de tipo causal (Hernández, Fernández y Baptista, 2006) y puesto que no se desprenden aplicaciones directas de esta experiencia, se trata de una investigación básica. Además se utilizarán estadísticos inferenciales para analizar los datos, de modo que se trata de un estudio de corte cuantitativo.

\section{Diseño de investigación}

El diseño de investigación es cuasi-experimental ya que se trabajó con un grupo establecido y los sujetos no fueron escogidos al azar. Asimismo, se aplicó una pre prueba y una post prueba sin tener grupo control, por tanto el gráfico del diseño de investigación es el siguiente:

\section{GE: $\mathrm{O}_{1} \quad \mathrm{X} \quad \mathrm{O}_{2}$}

\section{Población y muestra}

La población objeto de esta investigación está compuesta por todo el personal docente que se desempeña dentro de una institución educativa privada de Arequipa, sea que cumplan funciones administrativas o solamente docentes.

Para efectos de la recolección de datos se tomó como muestra a todo el personal docente, la cual está conformada por 17 varones (37\%) y 29 mujeres (29\%). Asimismo, la edad media de los participantes fue de 34 años con una desviación estándar de \pm 8.5 , dentro de un rango de 26 a 55 años. 
Para seleccionar a la muestra se utilizó un tipo de muestreo no probabilístico de grupos intactos, la cual fue tomada en cuenta por un criterio de conveniencia.

\section{Técnicas e instrumentos}

Se aplicó un Taller de coaching para los docentes de una institución educativa privada local, donde experimentarán los factores que generan relaciones positivas y constructivas, se revisará la manera de comunicarse y de gestionar los conflictos que en ocasiones se desencadenan. Utilizando para ello, los conocimientos y dinámicas del coaching, con el fin mejorar las relaciones interpersonales, de modo que sea valorada objetiva y cuantitativamente a través del incremento esperado en las puntuaciones de satisfacción de las relaciones interpersonales. Para evaluar esta variable se utilizará el Cuestionario de Satisfacción en las Relaciones Interpersonales en el Trabajo, tomado de Silva et al. (2008) que fue utilizado originalmente en España. Este instrumento consta de 13 ítems dispuesta en una escala de Likert de 7 alternativas que van desde muy insatisfecho hasta muy satisfecho. La prueba es unidimensional y en muestras españolas cuenta con niveles de validez y confiabilidad aceptables $(\alpha>$.70).

\section{Resultados}

Ahora se presenta el procesamiento de la información. Primero se realiza el análisis descriptivo y luego el comparativo y finalmente el análisis de regresión como pruebas de hipótesis para valorar los datos pre y post test. En la primera tabla se tiene los valores descriptivos de la variable de estudio: la satisfacción de las relaciones interpersonales pre y post test.

Tabla 1.

Valores descriptivos

\begin{tabular}{|l|r|r|}
\hline & $\begin{array}{c}\text { Satisfacción de } \\
\text { las RI Pre test }\end{array}$ & $\begin{array}{c}\text { Satisfacción de } \\
\text { las RI Post test }\end{array}$ \\
\hline Media & 49.565 & 57.760 \\
\hline Mediana & 50.500 & 60.000 \\
\hline Moda & 42,00 & 61.00 \\
\hline Desv. típ. & 9.620 & 9.566 \\
\hline Varianza & 92.562 & 91.519 \\
\hline Asimetría & -.745 & -5.283 \\
\hline Curtosis & .779 & 31.023 \\
\hline Rango & 41.00 & 65.00 \\
\hline Mínimo & 23.00 & 0.00 \\
\hline Máximo & 64.00 & 65.00 \\
\hline
\end{tabular}

Para valorar el efecto del taller de coaching se aplicó en primer lugar la prueba paramétrica $\mathrm{t}$ de student, con la finalidad de comparar la pre prueba y la post prueba, de modo que si se hallan diferencias significativas entre las medidas del pre-test y el post-test, esto sugeriría que el taller de coaching ha sido efectivo. Como se puede apreciar en la Tabla 2, las diferencias entre el pres test y el post test medido a través de la satisfacción de las relaciones interpersonales en el trabajo, son significativas ( $\mathrm{p}<$ o.ooo) y comprende 8.195 puntos de diferencia. 
Tabla 2.

Comparaciones de medias mediante la t student

\begin{tabular}{|l|c|c|c|c|c|c|c|c|}
\hline & $\mathrm{t}$ & $\mathrm{gl}$ & $\begin{array}{c}\text { Sig. } \\
\text { (bilateral) }\end{array}$ & $\begin{array}{c}\text { Diferencia } \\
\text { de medias }\end{array}$ & $\begin{array}{c}\text { Desviación } \\
\text { estándar }\end{array}$ & \multicolumn{2}{|c|}{$\begin{array}{c}\text { Erro } \\
\text { típico }\end{array}$} & \multicolumn{2}{|c|}{$\begin{array}{c}95 \% \text { Intervalo de } \\
\text { confianza para la } \\
\text { diferencia }\end{array}$} \\
\cline { 6 - 9 } & & & & & & & Inferior & Superior \\
\hline $\begin{array}{l}\text { Satisfacción } \\
\text { RI Pre-test }\end{array}$ & 34.941 & 46 & 0.000 & 49.565 & 9.620 & 1.418 & 46.708 & 52.422 \\
\hline $\begin{array}{l}\text { Satisfacción } \\
\text { RI Post-test }\end{array}$ & 40.950 & 46 & 0.000 & 57.760 & 9.566 & 1.410 & 54.919 & 60.601 \\
\hline
\end{tabular}

Estos datos confirman la efectividad del taller de coaching, pero, dando cumplimiento a nuestro segundo objetivo, se hicieron diversas comparaciones en función de las variables sociolaborales. Por ejemplo, se compararon los valores de la satisfacción de las relaciones interpersonales en función del sexo. En la Tabla 3 se presentan los resultados, donde se aprecia que no hay diferencias significativas entre varones y mujeres, ni antes ni después del taller de coaching $(\mathrm{p}<0.134)$.

Tabla 3.

Comparaciones de medias mediante la t student según sexo

\begin{tabular}{|l|l|l|r|r|r|r|r|r|}
\hline & Sexo & $\mathrm{N}$ & Media & Desviación típ. & $\begin{array}{c}\text { Error típ. de la } \\
\text { media }\end{array}$ & $\mathrm{t}$ & $\mathrm{gl}$ & $\mathrm{p}$ \\
\hline $\begin{array}{l}\text { Satisfacción } \\
\text { RI Pre-test }\end{array}$ & Varón & 17 & 49.235 & 11.138 & 2.701 & -0.165 & 44 & 0.869 \\
\cline { 2 - 9 } & Mujer & 29 & 49.758 & 8.818 & 1.637 & & & \\
\hline $\begin{array}{l}\text { Satisfacción } \\
\text { RI Post-test }\end{array}$ & Varón & 17 & 59.941 & 1.951 & 0.473 & 1.536 & 44 & 0.134 \\
\cline { 2 - 10 } & Mujer & 29 & 56.482 & 11.846 & 2.199 & & & \\
\hline
\end{tabular}

También se hicieron comparaciones en función del grado de instrucción de los docentes, encontrándose que no hay diferencias significativas entre los profesores, para ello se utilizó la prueba paramétrica del Análisis de varianza. No se encontraron diferencias significativas, aunque las diferencias en el pre-test casi llegan al nivel de significancia estadística $(\mathrm{p}<0.074)$ (ver Tabla 4). 
Tabla 4.

Análisis de varianza en función del grado de instrucción

\begin{tabular}{|l|l|r|r|r|r|r|}
\hline & & \multicolumn{1}{c}{$\begin{array}{c}\text { Suma de } \\
\text { cuadrados }\end{array}$} & gl & \multicolumn{1}{c|}{$\begin{array}{c}\text { Media } \\
\text { cuadrática }\end{array}$} & F & Sig. \\
\hline \multirow{2}{*}{$\begin{array}{l}\text { Satisfacción RI } \\
\text { Pre-test }\end{array}$} & Inter-grupos & 293.426 & 1 & 293.426 & 3.334 & 0.074 \\
\cline { 2 - 7 } & Intra-grupos & 3871.878 & 44 & 87.997 & & \\
\cline { 2 - 7 } & Total & 4165.304 & 45 & & & \\
\hline \multirow{2}{*}{$\begin{array}{l}\text { Satisfacción RI } \\
\text { Post-test }\end{array}$} & Inter-grupos & 210.560 & 1 & 210.560 & 2.370 & 0.130 \\
\cline { 2 - 7 } & Intra-grupos & 3907.809 & 44 & 88.813 & & \\
\cline { 2 - 7 } & Total & 4118.369 & 45 & & & \\
\hline
\end{tabular}

Al hacer las comparaciones en función del tipo de profesión, se tiene que, tampoco hubo diferencias significativas entre los profesores, ni antes ni después de la aplicación del taller de coaching. La Tabla
5 resume los resultados del análisis de varianza, donde los valores estuvieron muy lejos de ser significativos, por tanto las diferencias encontradas no tienen validez estadística.

Tabla 5.

Análisis de varianza en función de la profesión

\begin{tabular}{|l|l|r|r|r|r|r|}
\hline & \multicolumn{1}{|c|}{$\begin{array}{c}\text { Suma de } \\
\text { cuadrados }\end{array}$} & gl & $\begin{array}{c}\text { Media } \\
\text { cuadrática }\end{array}$ & F & \multicolumn{1}{c|}{ Sig. } \\
\hline \multirow{2}{*}{$\begin{array}{l}\text { Satisfacción RI } \\
\text { Pre-test }\end{array}$} & Inter-grupos & 384.333 & 2 & 192.166 & 2.185 & 0.124 \\
\cline { 2 - 7 } & Intra-grupos & 3780.970 & 43 & 87.929 & & \\
\cline { 2 - 8 } & Total & 4165.304 & 45 & & & \\
\hline $\begin{array}{l}\text { Satisfacción RI } \\
\text { Post-test }\end{array}$ & Inter-grupos & 258.300 & 2 & 129.150 & 1.438 & 0.248 \\
\cline { 2 - 8 } & Intra-grupos & 3860.069 & 43 & 89.769 & & \\
\cline { 2 - 8 } & Total & 4118.369 & 45 & & & \\
\hline
\end{tabular}

En la Tabla 6 se tiene el análisis de varianza en función de la ocupación de los profesores. $\mathrm{Al}$ igual que en los casos anteriores, no se aprecian diferencias significativas, pero las diferencias en el pre test se acercan mucho a la significancia estadística $(\mathrm{p}<0.064)$. 
Tabla 6.

Análisis de varianza en función de la ocupación

\begin{tabular}{|l|l|r|r|r|r|r|}
\hline & \multicolumn{1}{|c|}{$\begin{array}{c}\text { Suma de } \\
\text { cuadrados }\end{array}$} & \multicolumn{1}{c|}{ gl } & $\begin{array}{c}\text { Media } \\
\text { cuadrática }\end{array}$ & \multicolumn{1}{c|}{ F } & \multicolumn{1}{c|}{ Sig. } \\
\hline \multirow{2}{*}{$\begin{array}{l}\text { Satisfacción RI } \\
\text { Pre-test }\end{array}$} & Inter-grupos & 499.777 & 2 & 249.888 & 2.931 & 0.064 \\
\cline { 2 - 8 } & Intra-grupos & 3665.526 & 43 & 85.244 & & \\
\cline { 2 - 8 } & Total & 4165.304 & 45 & & & \\
\hline \multirow{3}{*}{$\begin{array}{l}\text { Satisfacción RI } \\
\text { Post-test }\end{array}$} & Inter-grupos & 295.260 & 2 & 147.630 & 1.660 & 0.202 \\
\cline { 2 - 8 } & Intra-grupos & 3823.108 & 43 & 88.909 & & \\
\cline { 2 - 8 } & Total & 4118.369 & 45 & & & \\
\hline
\end{tabular}

En la Tabla 7, se valoran las diferencias diferencias encontradas no fueron signien función del área de trabajo, pero las ficativas.

Tabla 7 .

Análisis de varianza en función del área de trabajo

\begin{tabular}{|l|l|r|r|r|r|r|}
\hline & & \multicolumn{1}{c|}{$\begin{array}{c}\text { Suma de } \\
\text { cuadrados }\end{array}$} & gl & \multicolumn{1}{c|}{$\begin{array}{c}\text { Media } \\
\text { cuadrática }\end{array}$} & F & Sig. \\
\hline \multirow{2}{*}{$\begin{array}{l}\text { Satisfacción RI } \\
\text { Pre-test }\end{array}$} & Inter-grupos & 197.434 & 3 & 65.811 & 0.696 & 0.559 \\
\cline { 2 - 7 } & Intra-grupos & 3967.869 & 42 & 94.473 & & \\
\cline { 2 - 7 } & Total & 4165.304 & 45 & & & \\
\hline \multirow{2}{*}{$\begin{array}{l}\text { Satisfacción RI } \\
\text { Post-test }\end{array}$} & Inter-grupos & 68.924 & 3 & 22.974 & 0.238 & 0.869 \\
\cline { 2 - 7 } & Intra-grupos & 4049.445 & 42 & 96.415 & & \\
\cline { 2 - 7 } & Total & 4118.369 & 45 & & & \\
\hline
\end{tabular}

Para finalizar con las comparaciones, se hallados tampoco resultaron significativos realizó una última comparación en función (ver Tabla 8). del cargo de los profesores, pero los valores

Tabla 8.

Análisis de varianza en función del cargo

\begin{tabular}{|l|l|r|r|r|r|r|}
\hline & \multicolumn{1}{|c|}{$\begin{array}{c}\text { Suma de } \\
\text { cuadrados }\end{array}$} & gl & \multicolumn{1}{c|}{$\begin{array}{c}\text { Media } \\
\text { cuadrática }\end{array}$} & F & Sig. \\
\hline $\begin{array}{l}\text { Satisfacción RI } \\
\text { Pre-test }\end{array}$ & Inter-grupos & 483.913 & 3 & 161.304 & 1.840 & 0.154 \\
\cline { 2 - 7 } & Intra-grupos & 3681.390 & 42 & 87.652 & & \\
\cline { 2 - 7 } & Total & 4165.304 & 45 & & & \\
\hline $\begin{array}{l}\text { Satisfacción RI } \\
\text { Post-test }\end{array}$ & Inter-grupos & 419.096 & 3 & 139.698 & 1.586 & 0.206 \\
\cline { 2 - 7 } & Intra-grupos & 3699.272 & 42 & 88.077 & & \\
\cline { 2 - 7 } & Total & 4118.369 & 45 & & & \\
\hline
\end{tabular}




\section{Discusión}

El coaching es un proceso mediante el cual se acompaña a la persona para que pueda desarrollar sus metas y destrezas, poniendo en práctica sus habilidades, dentro del curso de ciertos métodos y valores establecidos que actúan como motivadores promoviendo las emociones positivas y ciertas habilidades sociales (Wise y Avendaño, 2013). Por ello, se dice que el coaching transforma a la persona, en cuanto le permite desarrollar una nueva visión (más optimista) de sí mismo, de su trabajo y el mundo que le rodea, proyectándose a futuro y comprometiéndose con todo lo que hace.

El coaching pone en el centro de todo a la persona y su mundo interior como exterior, vale decir su vida emocional y sus cogniciones así como su comportamiento (Palacios, 2008). En el coaching por tanto se establece un vínculo de confianza entre el coach y el coachee, y para entender esta relación, existen diversos marcos explicativos como la teoría sociocultural de Vigotsky a partir de la zona de desarrollo próximo, las teorías de la psicología positiva, el enfoque sistémico, la perspectiva neurocientífica (sobre todo desde la programación neurolingüística), etc.

El profesorado, en sus múltiples facetas, debe incluir el coaching tanto en la formación docente como en su quehacer pedagógico (Berga y Dunn, 1987). Para ello, se debe promover una visión de formación continua y autoexigencia (ISPA, 2002). Más aún si las condiciones laborales de los profesores les exponen a diversos factores de riesgo psicosocial (Fernández, 2002).
En nuestro medio por ejemplo, se ha visto cómo el estrés docente afecta su desempeño de manera diferenciada y que en parte esto se debe a las pésimas relaciones interpersonales que se establecen entre los profesores (Arias, 2013a).

Es importante por ello, gestionar adecuadamente el quehacer docente (Goncalves, 1997), para ello, se debe tener presente que las relaciones humanas en el trabajo son esenciales (Wieman, 2011). Diversos estudios nos ilustran acerca de cómo las relaciones interpersonales se relacionan con el clima organizacional, la satisfacción laboral, el liderazgo, el trabajo en equipo y la eficiencia laboral (Tse et al., 2008; Sierra et al., 2010).

Pensamos que a partir de las relaciones interpersonales en el trabajo se puede generar mayor bienestar en el profesor (Holmes, 2014), ya que así lo demuestran también diversos estudios locales (Arias, 2013a). En ese sentido hay que considerar que si los profesores no llevan adecuadas relaciones humanas, los estudiantes tampoco las desarrollarán, afectando un aspecto tan importante en sus vidas como es el aprender a convivir (Cueva y Vilca, 2013). También tendría un efecto muy favorable en su desempeño profesional al gestionar su talento en el entorno laboral (Alles, 2009).

En ese sentido, nuestro trabajo buscó valorar el efecto de un taller de coaching en las relaciones interpersonales de los profesores de una institución educativa local. Hay que tener en cuenta que el coaching ha sido muy poco estudiado en Arequipa y el Perú, por lo que el presente trabajo tiene un importante valor teórico y hace un 
aporte metodológico, al usar una metodología empírica y experimental para abordar esta temática. Solo pudimos encontrar un trabajo similar en Trujillo pero que aplica un taller de habilidades sociales para mejorar las relaciones interpersonales de los profesores, con resultados muy favorables (Rosales y Valverde, 2008).

Los resultados de la presente investigación también lo fueron, en la medida que se encontraron diferencias significativas antes y después de la aplicación del taller de coaching en la satisfacción de las relaciones interpersonales de los profesores participantes, aunque no encontramos diferencias significativas en función de las variables sociolaborales (sexo, grado de instrucción, profesión, ocupación, cargo, etc.). De este modo, nuestro trabajo reviste de importancia, al demostrar de manera objetiva y cuantificable, los beneficios del taller de coaching, sobre las relaciones interpersonales, que tienen íntimas vinculaciones con las habilidades sociales (Vallés, 1995), la inteligencia emocional (Goleman, 200o), el liderazgo (Jay, 2004), etc.

En ese sentido, se tiene la seguridad que esta investigación puede servir de modelo para muchas otras, ya que los resultados no son generalizables a otras instituciones educativas. De hecho, hace falta muchas más confirmaciones a través de la investigación de los beneficios del coaching, para lo cual deben controlarse mejor aspectos tales como el enfoque del coach, el rol del coach, la gestión de la institución educativa, su diagnóstico situacional y organizacional, las técnicas y métodos empleados, etc.
Esperamos que con esta experiencia, el coaching sea cada vez más objeto de estudio de los educadores, de los psicólogos, de los administradores y de todas las personas involucradas con sus principios, métodos y enfoques; pues muchas veces es visto solo como una práctica motivadora y no transformadora. Hace falta también valorar los efectos del programa a largo plazo, para lo cual hubiera sido necesaria una metodología diferente y que no hemos podido implementar, pues tales pretensiones están fuera de nuestros objetivos por esta oportunidad. Pero en futuras ocasiones, ahondaremos más y más en los aspectos antes mencionados, ya sean teóricos o metodológicos.

\section{Referencias}

Alles, M. A. (2009). "Construyendo talento". Buenos Aires: Granica.

Arias, W. L. (2008). "Fundamentos del aprendizaje”. Arequipa: Vicarte.

Arias, W. L. (2013a). Relaciones humanas en profesores de instituciones educativas públicas de Arequipa. "Revista de Investigación ULaSalle”, 2(1), 5-14.

Arias, W. L. (2013b). Clima organizacional en ocho empresas de Arequipa. "Illustro", 4, 39-56.

Arias, W. L. \& Jiménez, N. A. (2013a). Síndrome de burnout en docentes de educación básica regular de Arequipa. “Educación”, 22(42), 53-76.

Arias, W. L. \& Jiménez, N. A. (2013b). Relaciones humanas en mypes de 
Arequipa. "Contabilidad y Negocios”, 8(16), 48-6o.

Arias, W. L.; Montes, I. \& Masías, M. A (2014). El modelo demanda-control de Karasek y su relación con la creatividad en profesores de nivel primario de Arequipa. "Revista de Psicología”, 16(1), 64-77.

Arias, W. L.; Zavala, S. \& Bernilla, A. (2014). Calidad de la gestión educativa y rendimiento académico en la Institución educativa Arequipa. "Apuntes de Ciencia \& Sociedad", 4(1), 102-112.

Arqueros, M. T. (2009). El coaching en las organizaciones. "IV Jornadas Internacionales Mentoring \& Coaching”, 23-25 de noviembre, Madrid.

Ayuso, J. L.; Calzada, R.; Díaz, E.; Díaz, J.; González, G.; Gorroño, I.; Rivera, E.; Sierra, M. \& Yome, E. (2010). Coaching en equipos. Herramientas de diagnóstico. "Cuadernos de Coaching", 4, 16-18.

Barrientos, R. (2015). El liderazgo sí importa: aportes para una mejor comprensión de la dirección escolar. "Educationis Momentum”, 1(1), 83-119.

Bergan, J. R. \& Dunn, J. A. (1987). "Biblioteca de la psicología de la educación”. México: Editorial Limusa, S. A.

Berganza, A. \& Escribano, M. P. (2004). Coaching y mentoring como metodologías de desarrollo en la sorganizaciones. "Capital Humano”, 174, 27-34.
Berne, E. (1998). "Psicología de relaciones humanas". New York: Editorial Grove Press.

Bisquera, J. (2003). "Relaciones interpersonales". Madrid: Editorial McGraw-Hill.

Bunge, M. (1988). "La ciencia, su filosofía y su método”. Buenos Aires: Ariel.

Cueva, R. \& Vilca, A. (2013). "Relaciones interpersonales de los docentes y habilidades sociales en los estudiantes del quinto de secundaria de las instituciones educativas de los distritos de Aplao y Corire. Provincia de Castilla”. (Tesis para optar el título profesional de educador). UNSA, Arequipa.

Díaz, A. (2005). "La evaluación de las relaciones con los compañeros”. Madrid.

Díaz, L. E. (2005). Relaciones laborales especiales: las empresas de trabajo temporal y las cooperativas. ¿Qué pueden hacer los sindicatos? "Revista de Ciencias Sociales”, 11(1), 131-148.

Escribano, S. (2009). "Neurocoaching entre la ciencia y la vida”. Buenos Aires: Alienta.

Fernández, M. (2002). "Realidad psicosocial del maestro de primaria”. Lima: Fondo Editorial de la Universidad de Lima.

Fernández, M. (2009). La evaluación de desempeño, la percepción de justicia y las reacciones de los empleados. “Contabilidad y Negocios", 4(8), 42-48. 
Fingerman, G. (1980). "Relaciones humanas. Fundamentos psicológicos y sociales". Buenos Aires: El Ateneo.

Goleman, D. (200o). "La inteligencia emocional”. Barcelona: Kairós.

Goleman, D. (2007). "La salud emocional”. Barcelona: Kairós.

Goncalves, O (1997). "El proceso organizacional". Barcelona: Paidos

Gorrochotegui-Martell, A.; Vicente-Mendoza, I \& Torres-Escobar, G. (2014). Evaluación del proceso de coaching en directivos y su impacto en el clima escolar. "Educación y Educadores”, 17(1), 111-131.

Hernández, J. (2004). “Las relaciones interpersonales”. Malaga: Aljibe.

Hernández, R.; Fernández, C. y Baptista, P. (2010). "Metodología de la Investigación”. México: McGraw-Hill.

Hochman, L. (2010). "La revolución de las relaciones personales". Barcelona: Ediciones Urbano.

Holmes, E. (2014). "El bienestar de los docentes". Madrid : Narcea.

ISPA (2002). "Programa de formación continua de Docentes en Servicio". Arequipa: ISPA.

Jay , E. (2004). "Coaching y liderazgo". Madrid: Aguilar.

Jiménez, R. (2012a). Coaching en el desarrollo profesional del docente. Fases formativas y procesos metodológicos de investigación. "Revista Electrónica Interuniversitaria de Formación del Profesorado", 15(4), 101-112.

Jiménez, R. (2012b). La investigación sobre coaching en formación del profesorado: Una revisión de estudios que impactan en la conciencia sobre la práctica docente. "Profesorado. Revista de Curriculum y formación del Profesorado", 16(1), 238-255.

Larez, J. H. (2008). El coaching educativo como estrategia para potenciar el éxito durante la etapa de desarrollo y culminación del trabajo especial de grado. "Sapiens", 9(2), 219-234.

Kilburg, R. R. (2001). Facilitating Intervention Adherence in Executive Coaching. "Consulting and Psychology Journal: Practice and Research", 53(4), 251-267.

Kilpatrick, A. L. (1994). "Evaluating Training Programs: The four levels". San Francisco: Berrett-Koehler.

Lugo, E. (2004). "Las relaciones interpersonales en el departamento administrativo de la UPEL". (Tesis para optar el título profesional de Administrador). Universidad Pedagógica Libertador Maturin Monagas.

Maisel, E. (2005). “Coaching para el creativo que hay dentro de ti”. Mexico: Obelisco.

Mamani, M. (2006). Clima institucional y habilidades sociales. Arequipa.

Matalinares, M. L.; Sotelo, L.; Sotelo, N.;Arenas, C.; Díaz, G.; Dioses, A.; Yaringaño, J.; 
Murata, R. \& Pareja, C. (2009). Juicio moral yvalores interpersonales en estudiantes de secundaria de las ciudades de Lima y Jauja. "Revista de Investigación en Psicología", 12(2), 115-132.

Moreno, B.; Álvarez, M. \& Inojosa, Y. (2013). Incidencia de la metodología coaching como fundamento en la producción de conocimientos y desarrollo de la calidad del investigador en la Universidad Pedagógica Experimental Libertador. "Razón y Palabra", 83, 1-22.

Nava, A. \& Mena, M. (2012). Coahing y competencias cognitivas de los directores de escuelas de las universidades públicas binacionales. "Gestión \& Gerencia”, 6(1), 109-132.

O’Connor, J. \& Lages, A. (2010). “Coaching con PNL. Guía para obtener lo mejor de ti mismo y de los demás”. Barcelona: Urano

Orduña, A. (2006). "La influencia de las relaciones interpersonales, en el clima organizacional de estadísticas y encuestas, coordinación INEGI de Pachuca-Hidalgo-México". (Tesis para optar el grado de Maestro), México.

Palacios, L. E. (20o8). "El concepto de persona”. Madrid: Rialp.

Passmore, J. (2007). An integrating model for executive coaching. "Consulting and Psychology Journal: Practice and Research", 59(1), 68-78.
Pérez, M. (2001). “Afectos, emociones y relaciones en la escuela: análisis de cinco situaciones”. Barcelona: Grao.

Portugal, L. (200o). "Psicología social”. Arequipa: UNSA.

Ripoll, P.; González-Navarro, P.; Zornoza, A. \& Orengo, V. (2004). La influencia de los procesos de interacción grupal y el medio de comunicación sobre la eficacia de los grupos de trabajo. "Revista Latinoamericana de Psicología”, 36(2), 195-208.

Rodríguez, A.; Esteban, R. M.; Aranda, R.; Blanchard, M.; Dominguez, C.; González, P.; Romero, P.; Sanz, E.; Mampaso, A.; Vitón, M. J. \& Messina, C. (2011). Coaching reflexivo entre iguales en el Practitum de la formación de maestros. "Revista de Educación", 355, 355-379.

Rosales, D. y Valverde, C. (2005). Aplicación del programa de habilidades sociales ACAT para contribuir mejorar las relaciones interpersonales en los docentes de la institución educativa GUE José Faustino Sánchez Carrión de Trujillo. (Tesis para optar el grado de Maestro). Trujillo.

Rota, C.; Reynolds, N. \& Zanasi, C. (2012). The influence of organizational climate on sustainable relationships between organization and employees. The KION case study. "Advances in Management \& Applied Economics", 2(4), 125-140. 
Sánchez, M. (2015). Efectos de un taller basado en la pedagogía del amor y la ternura para mejorar la disciplina en estudiantes de primer grado de primaria de una escuela arequipeña. "Educationis Momentum", 1(1), 61-81.

Saporito, T. J. (1996). Business-linked excutive development. Coaching Senior Executives. "Consulting and Psychology Journal: Practice and Research", 48(2), 96-103.

Seligman, M. E. P. (2006). "La auténtica felicidad”. Buenos Aires: Vergara.

Sierra, V.; Andrés, A.; Solanas, A. \& Leiva, D. (2010). Agreement in interpersonal perception as a predictor of group performance. "Psicothema", 22(4), 848-857.
Silva, M. M.; Santos, J. L.; Rodríguez, E. \& Rojo, C. H. (2008). "Relaciones humanas en la empresa”. Madrid: Paraninfo.

Valles, A. (1995). "Las habilidades sociales en el trabajo". Madrid: Editorial EOS.

Tse, H. H.; Dasborough, M. T. \& Ashanasy, N. M. (2008). A multi-level analysis of team climate and interpersonal Exchange relationships at work. "The Leadership Quarterly”, 19(2), 195-211.

Wiemann, M. (2011). "La comunicación en las relaciones interpersonales”. España: Aresta.

Wise, D. \& Avendaño, K. (2013). "Coaching para el liderazgo educativo”. Guatemala: USAID.

Recibido: 30-01-2016

Aceptado: 12-10-2016 